\title{
Mapping the literature of pediatric nursing: update and implications for library services $P$ EC
}

\author{
Carol L. Watwood, MLS, MPH, AHIP
}

See end of article for author's affiliation.

DOI: http://dx.doi.org/10.3163/1536-5050.104.4.005

\begin{abstract}
Objective: The purpose of this study was to identify core journals and other types of literature cited in four major pediatric nursing journals and to characterize coverage of these resources in major bibliographic databases. The study was part of the "Mapping the Literature of Nursing Project" of the Medical Library Association's Nursing and Allied Health Resource Section. It updates a similar analysis published in 2006 and determines whether citation patterns have changed over time.
\end{abstract}

Methods: Cited references from articles published in 4 pediatric nursing journals between 2011 and 2013 were collected. Cited journal titles were ranked according to number of times cited and analyzed according to Bradford's Law of Scattering and the 80/20 rule to identify the most frequently cited journals. Five databases were surveyed to assess the coverage of the most-often-cited journals. The most frequently cited non-journal sources were also identified.

Results: Journals were the most frequently cited sources, followed by books, government documents, Internet resources, and miscellaneous resources. Most cited sources were cited within ten years of their publication, which was particularly true for government documents and Internet resources. Scopus had complete coverage of the most frequently cited journals, whereas PubMed had nearly complete coverage.

Conclusions: Compared with the 2006 study, the list of top-cited journals referenced by pediatric nursing researchers has remained relatively stable, but the number of cited journal titles has increased. Book citations have declined, and Internet and government document references have increased. These findings suggest that librarians should retain subscriptions to frequently cited journal titles, provide efficient document delivery of articles from infrequently used journals, deemphasize but not eliminate books, and connect patrons with useful open-access Internet resources.

Keywords: Pediatric Nursing/Statistics and Numerical Data, Bibliometrics, Abstracting and Indexing as Topic/Statistics and Numerical Data, Periodicals as Topic/Statistics and Numerical Data

Pediatric nurses - who work primarily with infants, children, and adolescents-number about 362,000, slightly more than one-tenth of the 3.1 million registered nurses (RNs) in the United States [1]. Within this specialty, neonatal nurses work mostly in hospitals, and child or adolescent nurses work mainly with outpatients [1]. These nurses assess and

Sis

This article has been approved for the Medical Library Association's Independent Reading Program < http://www.mlanet. org/page/independent-reading-program $>$.

EC Supplemental Table 4 and Table 5 are available with the online version of this journal. monitor the attainment of developmental and cognitive milestones and incorporate both the child and family caregiver in their unit of care [2]. Pediatric nurses strive in age-appropriate ways to minimize the physical and psychological distress of children and their families. For example, a nurse may ask a child to point to the face on the Wong-Baker face scale that shows "how bad it hurts." Following the decline of childhood mortality due to infectious disease, pediatric illness increasingly has a large "behavioral, social, and educational component" [3]. Pediatric nurses are seeing sicker children with higher technology needs and families with complex needs, financial challenges, and lack of insurance [4]. 
In addition to disease processes, they deal with multicultural and family care; safety, health promotion, and environmental health; clinical teaching in community, school, and well-care settings; and home care [2]. Pediatric nurses also advocate for their vulnerable young patients at all levels of society.

Most US pediatric nurses are RNs, and many belong to the Society of Pediatric Nurses (SPN), which incorporates all levels of pediatric nursing [5]. The National Association of Pediatric Nurse Practitioners (NAPNAP) represents pediatric nurse practitioners (PNPs) and other pediatric advanced practice nurses, who usually have a master's degree or doctorate. The very first advanced practice nurses were PNPs who, in the mid-1960s, were taught to perform physical examinations, diagnose and treat children, and counsel families [6].

Pediatric nursing has a robust literature that can be analyzed to identify core journals, databases, books, websites, and other resources that should be collected by health sciences librarians. The latest edition of Pediatric Nursing: Scope and Standards of Practice calls for an increased emphasis on research, evidence-based practice, and clinical scholarship. Its standard 9, "Evidence-Based Practice and Research," states that " $t]$ he pediatric nurse integrates research findings into practice and, where appropriate, participates in the generation of new knowledge" [7]. Furthermore, SPN and NAPNAP actively promote research and evidence-based practice.

In 2006, Mary K. Taylor, AHIP, investigated the literature of pediatric nursing as part of the "Mapping the Literature of Nursing Project" of the Nursing and Allied Health Resources Section of the Medical Library Association [8]. Taylor examined cited references from articles published between 1998 and 2000 in three pediatric nursing journals. The purpose of the research was to identify core journals, to determine bibliographic coverage, and to influence database producers to improve access to nursing journals. Since Taylor's study, major changes have impacted nursing research. For example, a 2000 article introduced the growing Cochrane Collaboration to obstetric nurses after MEDLINE began adding Cochrane abstracts in 1999. The Cochrane Database of Systematic Reviews later became widely used by nurses [9]. Seamless linking to electronic journal articles was facilitated by the introduction of PubMed LinkOut in 2001 [10]. In 2003, the CINAHL database, soon after acquired by EBSCO, also enabled links to local library holdings [11]. Furthermore, Google
Scholar debuted in 2004, added open uniform resource locator (URL) links to library holdings in 2005, and quickly became used as a supplement to CINAHL and MEDLINE [12]. Due to such changes in the extent of literature available to pediatric nursing researchers and its ease of access, the purpose of the present study was to update Taylor's findings and to investigate the library resources most frequently used by today's pediatric nurse researchers.

\section{METHOD}

Four pediatric nursing journals were analyzed in this study: Journal of Pediatric Nursing, Pediatric Nursing, Journal for Specialists in Pediatric Nursing, and Journal of Pediatric Health Care. The first three titles were previously analyzed in the 2006 study [8]. The Journal of Pediatric Nursing is affiliated with the SPN, and Journal of Pediatric Health Care is the official publication of NAPNAP. The fourth title, Journal of Pediatric Health Care, an advanced practice journal, was included after consulting with nursing faculty. All four journals are Brandon/Hill titles that are indexed in both CINAHL and MEDLINE.

All cited references from articles published in the four journals from 2011 to 2013 were copied from the publisher websites into an Excel spreadsheet. Letters to the editor and brief items were excluded. Unlike the 2006 study [8], the present study included editorials, as many editorials now have extensive lists of references. Online-only articles were also included.

Cited references were assigned to one of 5 different formats: $1=$ journal, $2=$ book, $3=$ government document, $4=$ Internet resources, or $5=$ miscellaneous. This method was based on the protocol described in the "Mapping the Literature of Nursing and Allied Health Professions: Project Protocol" [13]. If a reference belonged to more than one format, the first number was used. Documents, books, and websites produced by US federal, state, or local government entities were coded " 3 ," as were publications of other national governments. The full titles of journals, books, government documents, Internet resources, and miscellaneous resources were recorded. If a journal's title had changed over time, the older title was updated to the most recent one. The National Library of Medicine Catalog, OCLC WorldCat, and Google (in descending order) were used as authorities for journal names. Minor citation errors were common, but virtually all faulty citations were corrected using CINAHL, PubMed, Google Scholar, 


\begin{tabular}{|c|c|c|c|c|c|c|}
\hline Cited format type & JSPN & $P N$ & $J P N$ & $J P H C$ & Total & Percentage \\
\hline Journal articles & 3,024 & 1,612 & 6,918 & 4,206 & 16,498 & $77.5 \%$ \\
\hline Books & 360 & 235 & 869 & 454 & 2,029 & $9.5 \%$ \\
\hline Government documents & 117 & 93 & 384 & 288 & 956 & $4.5 \%$ \\
\hline Internet & 134 & 188 & 576 & 417 & 1,398 & $6.6 \%$ \\
\hline Miscellaneous & 76 & 57 & 187 & 61 & 413 & $1.9 \%$ \\
\hline Total & 3,711 & 2,185 & 8,934 & 5,426 & 21,294 & $100.0 \%$ \\
\hline \multicolumn{7}{|c|}{$\begin{array}{l}J S P N=\text { Journal for Specialists in Pediatric Nursing. } \\
P N=\text { Pediatric Nursing. } \\
J P N=\text { Journal of Pediatric Nursing. } \\
J P H C=\text { Journal of Pediatric Health Care. }\end{array}$} \\
\hline
\end{tabular}

\section{Table 1}

Citation frequency of format types by source journal

or e-journal websites. Cited source titles were then ranked by format and total number of times cited.

Total cited references in the one-fifth most-cited journals were counted, as it was hypothesized, according to the $80 / 20$ rule or Pareto distribution [14], that $20 \%$ of the journals would account for $80 \%$ of the citations. In a separate analysis, Bradford's Law of Scattering was used to examine the cited journal titles. The ranked journal titles were divided into 3 zones, each containing one-third of the cited references. According to Bradford's law, the numbers of titles in the 3 zones should fall into a 1:n:n2 distribution. The number of titles per zone is squared to predict the number in the next zone, and the number of journals required to yield the same number of relevant references increases exponentially with each zone. Bradford's law exists in several formulations: in this study, $\mathrm{n}=5$, meaning that Zone 2 was expected to have 5 times as many titles as Zone 1 [15].

Five databases were analyzed for coverage of Zone 1 and Zone 2 journals: PubMed, CINAHL, PsycINFO, Web of Science Core Collection (a newer product incorporating Science Citation Index and Social Science Citation Index), and Scopus. The first four databases were used in the 2006 study [8], whereas Scopus was used instead of EMBASE in the present study due to a lack of access to EMBASE. In contrast to the 2006 study [8], the present study relied on journal title coverage lists provided by individual database producers and simply listed the degree of coverage as "yes," "no," or "selective."

\section{RESULTS}

A total of 21,292 citations of articles published in the 4 pediatric nursing journals between 2011 and 2013 were included in this analysis. The Journal of Pediatric Nursing, the Journal of Pediatric Health Care, the Journal for Specialists in Pediatric Nursing, and Pediatric Nursing accounted for $41.7 \%$, 25.5\%, 17.4\%, and $15.4 \%$ of these citations, respectively. Journal articles accounted for the majority of citations, followed by books, government documents, Internet resources, and miscellaneous resources (Table 1).

Most cited references in all 5 formats were cited within 10 years of their publication in the decade from 2004 to 2013 (Table 2). Cited government documents and Internet resources were mostly published after 2003 (79.2\% and 85.3\%, respectively). Most cited journal articles (65.1\%) were also published after 2003. Slightly less than half of cited books $(47.1 \%)$ were published after 2003 . The miscellaneous category contained the largest proportion of cited works older than 50 years (3.4\%).

A total of 2,330 unique journal titles were cited. Similar to the 2006 study [8], the 80/20 rule was borne out in the frequency of journal title citations: $78.3 \%$ of references came from the top $20 \%$ of journals, representing 466 titles. However, the distribution among Zones 1-3 did not follow that predicted by Bradford's law, which predicted a 33:195:975 distribution of journal titles. Although Zone 1 contained 33 titles (including the 4 source journals) cited between 64 and 1,268 times, Zone 2 contained 213 titles cited between 12 and 63 times, and Zone 3 contained 2,085 titles cited between 1 and 12 times (Table 3). Of the Zone 3 titles, 1,481 were cited only once.

Table 4 (online only) lists Zone 1 and Zone 2 journals and whether they were indexed in Scopus, PubMed, Web of Science Core Collection, or CINAHL Complete. Scopus had the most comprehensive coverage, indexing 100\% of Zone 1 


\begin{tabular}{|c|c|c|c|c|c|c|c|c|c|c|c|}
\hline \multirow[b]{2}{*}{ Publication year } & \multicolumn{2}{|c|}{ Journal articles } & \multicolumn{2}{|c|}{ Books } & \multicolumn{2}{|c|}{$\begin{array}{l}\text { Government } \\
\text { documents }\end{array}$} & \multicolumn{2}{|c|}{$\begin{array}{l}\text { Internet } \\
\text { resources }\end{array}$} & \multicolumn{2}{|c|}{ Miscellaneous } & \multirow{2}{*}{$\begin{array}{l}\text { Total } \\
\text { No. }\end{array}$} \\
\hline & No. & Percent & No. & Percent & No. & Percent & No. & Percent & No. & Percent & \\
\hline 2004-2013 & 10,747 & $65.1 \%$ & 956 & $47.1 \%$ & 757 & $79.2 \%$ & 1,193 & $85.3 \%$ & 259 & $62.9 \%$ & 13,912 \\
\hline 1994-2003 & 4,670 & $28.3 \%$ & 665 & $32.8 \%$ & 135 & $14.1 \%$ & 137 & $9.8 \%$ & 78 & $18.9 \%$ & 5,685 \\
\hline 1984-1993 & 783 & $4.7 \%$ & 211 & $10.4 \%$ & 14 & $1.5 \%$ & 2 & $0.1 \%$ & 33 & $8.0 \%$ & 1,043 \\
\hline 1974-1983 & 177 & $1.1 \%$ & 99 & $4.9 \%$ & 4 & $0.4 \%$ & 1 & $0.1 \%$ & 15 & $3.6 \%$ & 296 \\
\hline 1964-1973 & 56 & $0.3 \%$ & 57 & $2.8 \%$ & 3 & $0.3 \%$ & 0 & - & 4 & $1.0 \%$ & 120 \\
\hline Pre-1964 & 61 & $0.4 \%$ & 36 & $1.7 \%$ & 1 & $0.1 \%$ & 2 & $0.1 \%$ & 14 & $3.4 \%$ & 114 \\
\hline No Year & 3 & $0.02 \%$ & 5 & $0.2 \%$ & 42 & $4.4 \%$ & 63 & $4.5 \%$ & 9 & $2.1 \%$ & 122 \\
\hline Total & 16,497 & $99.9 \%^{*}$ & 2,029 & $99.9 \% *$ & 956 & $100.0 \%$ & 1,398 & $99.9 \%{ }^{*}$ & 412 & $99.9 \%{ }^{*}$ & 21,292 \\
\hline
\end{tabular}

Table 2

Citation frequency of format types by publication year periods

and Zone 2 journals. PubMed fully indexed $100 \%$ of Zone 1 journals and 97\% of Zone 2 journals. Web of Science Core Collection indexed $97 \%$ of Zone 1 journals and $91 \%$ of Zone 2 journals. CINAHL Complete indexed $94 \%$ of Zone 1 journals and $85 \%$ of Zone 2 journals. PsycINFO fully indexed 33\% of Zone 1 journals and 33\% of Zone 2 journals.

\section{DISCUSSION}

Journal articles accounted for more than threequarters of cited references $(77.5 \%)$ in major pediatrics nursing journals, which is a greater proportion of cited references than in the 2006 study $(71.8 \%)$ [8]. Furthermore, many more unique journal titles were represented in the present study than in the 2006 study $(2,331$ versus 1,273$)$. Whereas 33 journals were included in Zone 1 in the present study, only 18 journals were included in Zone 1 in the 2006 study [8]. However, it must be taken into account that the total number of cited references in the present study was also greater than that in the 2006 study (21,292 versus 9,769). The addition of the

\begin{tabular}{|c|c|c|c|c|c|}
\hline \multirow[b]{2}{*}{ Zone } & \multicolumn{2}{|c|}{ Journals } & \multicolumn{2}{|c|}{ References } & \multirow{2}{*}{$\begin{array}{c}\begin{array}{c}\text { Cumulative } \\
\text { total }\end{array} \\
\text { No. }\end{array}$} \\
\hline & No. & Percentage & No. & Percentage & \\
\hline 1 & 33 & $1.4 \%$ & 5,493 & $33.3 \%$ & 5,493 \\
\hline 2 & 213 & $9.1 \%$ & 5,498 & $33.3 \%$ & 10,991 \\
\hline 3 & 2,085 & $89.4 \%$ & 5,504 & $33.4 \%$ & 16,495 \\
\hline Total & 2,331 & $99.9 \% *$ & 16,495 & $100.0 \%$ & \\
\hline \multicolumn{6}{|c|}{ * Does not equal $100.0 \%$ due to rounding. } \\
\hline
\end{tabular}

Table 3

Cited journals and references by zone
Journal of Pediatric Health Care as a fourth source journal, the inclusion of editorials, and a possible trend toward longer lists of cited references might have contributed to this increase in number of cited references. The increasing number of journals cited highlights the increasing importance of fast, efficient document delivery to retrieve full-text articles that are not available locally.

The most-cited journals remained relatively stable over time: 14 of the 18 Zone 1 journals from the 2006 study [8] remained in Zone 1 in the present study. Only 9 of the top 33 journals were primarily nursing journals. Other top-cited journals included Pediatrics, the Journal of Pediatrics, the Journal of Pediatric Psychology, JAMA, the Journal of Adolescent Health, and the American Journal of Public Health. The Cochrane Database of Systematic Reviews, which can be considered both a journal and a database, was a new appearance in Zone 1. Pediatrics remained in the top position, indicating that reliable access to this publication should be a high priority for librarians supporting pediatric nursing research. Other publications of the American Academy of Pediatrics were also highly cited, including Pediatrics in Review, Bright Futures, and Red Book.

Books were cited less often in the present study than in the 2006 study [8] (9.5\% versus $21.3 \%$ of total citations). This decline in book citations mirrors a general decline in academic library book circulation [16]. The top 20 most highly cited non-journal resources included both print and e-books (Table 5, online only). Notably, more than half were print books that were unavailable online at the time of the study. Four books published by the National Academies Press were among the top 20 non-journal resources cited: The Future of Nursing: Leading 
Change, Advancing Health; Crossing the Quality Chasm: A New Health System for the 21st Century; To Err Is Human: Building a Safer Health System; and When Children Die: Improving Palliative and End-of-Life Care for Children and Their Families. These open-access publications should be highlighted in library research guides. The Diagnostic and Statistical Manual of Mental Disorders was also frequently cited. This resource is increasingly used by advanced-practice nurses and should be considered for site license.

The proportion of government documents increased from $3.0 \%$ in the 2006 study [8] to $4.5 \%$ in the present study. Many are freely available online, and $97 \%$ are "born digital" [17]. Two government documents, Expert Panel Report 3: Guidelines for the Diagnosis and Management of Asthma and Healthy People 2010, were among the top 20 non-journal sources cited. As expected, the percentage of Internet resources increased from $1.2 \%$ to $6.6 \%$ between the 2006 study [8] and the present study. Government documents and Internet resources were the cited format types that were most likely to be 10 years old or less.

As in the 2006 study [8], all types of cited sources dealt with a wide breadth of topics: pediatric nursing care; child development; families; research methodology; behavioral health; chronic diseases such as asthma, diabetes, and cancer; health promotion; scope of practice; public policy; and child health advocacy. Of the Zone 1 journals devoted to specific health issues, the following issues were represented: obesity, behavioral health, diabetes, asthma, allergy, nutrition, and cancer. Of the twenty most-cited non-journal resources, the topics of behavioral health, asthma, and palliative care were represented.

The citing of journals from other disciplines - such as JAMA, the New England Journal of Medicine, and the American Journal of Public Health-perhaps reflects the trend toward advanced education and certification of nurses, as pediatric nurses first led the way to advanced practice nursing in the mid-1960s. In 2010, the Institute of Medicine's Future of Nursing report argued that nurses should "practice to the full extent of their education and training," "achieve higher levels of education," and "be full partners, with physicians and other health care professionals, in redesigning health care in the United States" [18].

Database coverage was checked for Zone 1 and Zone 2 journals (representing the top two-thirds of cited journal references). Of the five databases,
Scopus reported the most complete coverage of Zone 1 and Zone 2 journals, but PubMed was a close second. PubMed is so widely used by nurse researchers, however, that a "chicken and egg" question arises: does PubMed index the journals that pediatric nurses need, or do they use them because they find them in PubMed? Web of Science may be considered a supplemental database for its broad science coverage and cited reference features. CINAHL Complete also had good coverage of both Zone 1 and Zone 2 journals and contains dissertations and other nursing materials not found in PubMed. Coverage of non-nursing journals varies widely in different versions of CINAHL, and libraries serving advanced practice pediatric nurses might prefer CINAHL Complete. PsycINFO had the least coverage of Zone 1 and Zone 2 journals and should not be used as a sole database; however, it has unique coverage of behavioral science resources.

Perhaps the widespread use of Google Scholar will change the rankings of scholarly journals over time. As yet, no decline in citation of well-established peer-reviewed journals was seen, but the increasingly "long tail" of journal titles may come partly from the use of Google Scholar. Also, new types of resources that do not fall easily into traditional categories are being cited. For example, the Cochrane Database of Systematic Reviews, which was a new appearance in Zone 1 in the present study, can be considered either a journal or a database. Also, point-of-care resources such as UpToDate appeared occasionally in citation lists and may be found more often in the future. In conclusion, citation mapping provides a valuable primary source of information on the changing use of library resources that can inform collection development decisions.

\section{ACKNOWLEDGMENTS}

The author gratefully acknowledges the contributions of Deanna Hanson, MSN, RN-BC, instructor of nursing at Western Kentucky University, and Daryl Watwood.

\section{REFERENCES}

1. US Department of Health and Human Services Health Resources and Services Administration. The registered nurse population findings from the 2008 National Sample Survey of Registered Nurses. Table 3-16. Primary patient age group cared for in principal nursing position, for 
hospital and nonhospital settings [Internet]. The Department; Sep 2010 [cited 24 Feb 2010]. <http://bhpr. hrsa.gov/healthworkforce/rnsurveys/rnsurveyfinal.pdf $>$. 2. Kocisko D, Rudd K, eds. Pediatric nursing: the critical components of nursing care. Philadelphia, PA: F.A. Davis; 2014.

3. Hockenberry MJ, Wilson D, eds. Wong's nursing care of infants and children. 9th ed. St. Louis, MO: Mosby; 2013. 4. Curry DM, Samawi Z. Preparing pediatric nurses for the 21st century: perceptions of nurse managers, nursing faculty, and staff nurses. J Pediatr Nurs. 2011 Dec;26(6): 599-603. DOI: http://dx.doi.org/10.1016/j.pedn.2011.09. 001.

5. Society of Pediatric Nurses [Internet]. [cited 24 Feb 2015]. < http://www.pedsnurses.org $>$.

6. National Association of Pediatric Nurse Practitioners. About: our business: history [Internet]. The Association [cited 24 Feb 2015]. < https://www.napnap.org/ourbusiness $>$.

7. National Association of Pediatric Nurse Practitioners, Society of Pediatric Nurses, American Nurses Association. Pediatric nursing: scope and standards of practice. 2nd ed. Silver Spring, MD: American Nurses Association; 2015.

8. Taylor MK. Mapping the literature of pediatric nursing. J Med Libr Assoc. 2006 Apr;94(2 suppl):E128-36. 9. Callister L, Hobbins-Garbett D. Cochrane pregnancy and childbirth database: resource for evidence-based practice. J Obstet Gynecol Neonatal Nurs. 2000 Mar-Apr; 29(2):123-8.

10. Nahin A, Canese K. LinkOut for libraries now available. NLM Tech Bull. 2001 Mar-Apr;319:3. 11. Chaskey G, Herrera G. MLA, 2003: one awesome event! Cinahlnews. 2003 22(1-2):8.

12. Grogg J, Ferguson C. OpenURL linking with Google Scholar. Searcher. 2005 Oct;13(9):39-46.

13. Nursing and Allied Health Resources Section. Mapping the literature of nursing and allied health professions: project protocol. Chicago, IL: Medical Library Association; 2010.

14. Nisonger TE. Serials in the core collection. the " $80 / 20$ rule" and core journals. Serials Libr. 2008;55(1-2):62-84. 15. Garfield E. Bradford's law and related statistical patterns. Current Comments. 1980 May 12;(19):476-83. (Available from: < http://www.garfield.library.upenn. edu/essays/v4p476y1979-80.pdf $>$. [cited 26 Apr 2016].) 16. Rose-Wiles L. Are print books dead? an investigation of book circulation at a mid-sized academic library. Tech Serv Q 2013;30(2):129-52.

17. US National Academy of Public Administration. Rebooting the Government Printing Office: keeping America informed in the Digital Age [Internet]. The Academy [cited 8 Apr 2015]. < http://www.gpo.gov/pdfs/ about/GPO_NAPA_Report_FINAL.pdf $>$.

18. Committee on the Robert Wood Johnson Foundation Initiative on the Future of Nursing at the Institute of Medicine; Robert Wood Johnson Foundation; Institute of Medicine (US). The future of nursing: leading change, advancing health [Internet]. Washington, DC: National Academies Press; 2011 [cited 24 Feb 2015]. < https://www. iom.edu/Reports/2010/The-Future-of-Nursing-LeadingChange-Advancing-Health.aspx $>$.

\section{AUTHOR'S AFFILIATION}

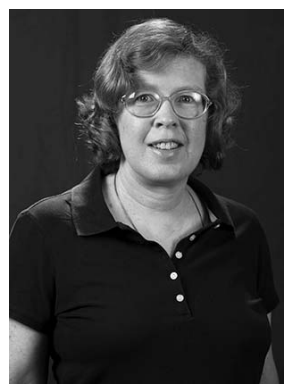

Carol L. Watwood, MLS, MPH, carol.watwood@wku.edu, Health Sciences Librarian/Associate Professor, Department of Library Public Services, Western Kentucky University, 1906 College Heights Boulevard \#11067, Bowling Green, KY 42101-1067

Received March 2016; accepted May 2016 